\title{
Theoretical Studies in Tonal Music: Thomas Adès \& Violin Concerto
}

\author{
Ceyla Ganioglu \\ Ankara Music and Fine Arts University, Faculty of Performing Arts, \\ Instrument Education Department, Strings Division, Ankara, Turkey \\ E-mail: ceylaganioglu@mgu.edu.tr \\ ORCID: 0000-0002-6772-7816
}

\begin{abstract}
This paper is going to evaluate "Concentric Paths" of Thomas Adès regarding different aspects such as the general characteristics, the motivation that makes Adès compose "Concentric Paths", the process of composing, and outstanding features.
\end{abstract}

\begin{abstract}
About the Cover
By taking a deep looking at its title, we can see that a path linearity. Moreover, it is for a kind of voyage that begins from one point and continues to roam among other points in time and space. The term "Concentricity", however, is in a contradiction with this teleological advance. In other words, it expresses loops, spirals, and circles. This Concerto of Violin and Chamber Orchestra of Adès, "Concentric Paths", was completed in 2005 and its manuscript is hold in Faber Music Ltd. 74/77 Great Russel St. London as Faber Music is also its original publisher in 2010 at London (May).

Each myth constitutes of, more or less, similar aspects like the Sun, the Earth, and the Stars. Planets are moving around the Sun while day and night are chasing each other. Then, people are added to this system with their daily life, their hearts, and their rhythms. On the cover of Violin Concerto of Adès, this mythic touch can be sensed. In the Star Atlas, 1660, "The Harmony of the Universe" can be seen. This same "The Harmony of the Universe" can also be seen on the cover of the original score for Violin Concerto of Adès. Therefore, one can say that the cover simply tries to make people feel what music is about and what music tells us about our lives.
\end{abstract}

Keywords: Thomas Adès \& Violin Concerto

DOI: $10.7176 / \mathrm{JSTR} / 7-03-03$

\section{Overview of the Violin Concerto}

The Violin Concerto was recorded in April 2007 in London's Barbicon Centre. The Concerto was accompanied by Chamber Orchestra of Europe. It was scored for Beethovenian forces with two percussionist. For the general characteristics, instrumentation is as follows; 2 flutes, 2 oboes, 2 clarnets ( 1 in $\mathrm{Bb}, 2$ in $\mathrm{A}$ ), 2 bassoons, 3 horns in $\mathrm{F} 2$ trumpets in $\mathrm{C}$, trombone, tuba, timpani, percussions (2players), strings, + doublebasses: all tune to lo Bb if possible. In terms of timpani, 5 drums are ideal with the condition of minimum 4. For percussion; Largebassdrums (quasilow A) and additionally, 2 lowdrums (e.g. lowtom-tom, tenor drum), and optionally all on bassdrum. Lowwooddrum - either a lowtom-tom with balsewood fitted exactly over the head or a large logdrum with single pitch, side drum with additional 3 low drums of different sizes (e.g deep military drums) and with optional side drum kits bass drum moving around the head to achieve different pitches, large - tam-tam (quasilow A) (labelled tam-tam 4), 3 extra tam-tams (small, medium, medium, large) -optional(labelled 1-3), clashed cymbals, suspendedcymbal, small bongo, metal guero, woodguero, lowwoodblock, largestraightcowbell (two instruments ad lib.), small orsquare tin - not sonorous (two instruments ad lib.), metal blockorlargeanvil. Furthermore, its duration is 20 minutes and was performed first on September 4, 2005, Berlin, while soloist was Anthony Marwood while the subsequent performance will 
be hold on 10th February 2006 as Anthony Marwood is soloist and Thomas Adès is conductor with Los Angeles Philarmonic Orchestra.

These are the works that were composed around the same period; Op.22 - The tempest (2003-2004), Op.22a - Scenes from the Tempest (2004), Court Studies from the Tempest (2005), 3 Studies from Couperin (2006). These, furthermore, are works in related styles; Arcadania (1994), Piano Quintet (2000), Court Studies from the Tempest (2005), and The Four Quarters (2010). Lastly, works by other composers in relevance with the Violin Concerto are as follows; Concertos of Berg and Ligeti, The Forms used by Mozart in the Idomeneo Ballet and in so many of his concertos, Automne à Varsovie by Ligeti, and Sonata for Solo Violin in g minor (Presto) by J.S. Bach (Mangum).

\title{
Composition History
}

\section{Brief Information of the composer, Thomas Adès}

Thomas Adès was born in London, England in 1 March 1971. His full name is Thomas Joseph Edmund Adès. He is a British composer, conductor, and pianist. He has a diversified works range from pieces to operas. This specialty makes him as one of the most-skilled music artist of his generation. Furthermore, he was trained as a pianist at the Guildhall School in London. He then attended King's College, Cambridge. Although he is famous as a composer, his first fame came for his virtuoso pianoplaying. He began to write music in 1990s and Powder Her Face, 1995 which is about a 20th-century divorce scandal, attracted international attention like his other work Asyla, 1997. As a pianist, including solo release Piano in 2000, he appeared on various recordings. Adès, in terms of his skills, is one of the most compelling and consistant composer of his generation. Starting by 2005 he did live four recordings which are composed by him over a three years period. He is considers as one of the leading composers and his Violin Concerto established itself rapidly as on of the few contemporary concertos in frequent rotation.

\section{Anthony Marwood and the Violin Concerto}

Anthony Marwood asked Thomas Adès for a concerto in 1995. However, Thomas Adès was able to finish this concerto in 2005, 10 years later. Anthony Marwood claimed that it is a little complex and difficult as well when he firstmet the score. His comment on this issue is;

\begin{abstract}
“...physically possible. It went from the highest point, way off the fingerboard at the top, cascading down in broken ninths to the bottom, then up again, then down again, and so on, very fast, with the journey being slightly shortened each time, till it finally broke up. I couldn't believe my eyes. I decided not to answer the question of whether it was possible - I just decided it would be, somehow. I was determined not to be the man who is laughed at by posterity, like someone saying the Brahms concerto was too hard."(Church).
\end{abstract}

\section{Analysis of the Violin Concerto}

The Violin Concerto has widen the hidden story into its orbits through other forms such as baroque style. Adès includes direct contact with the particularity of constructivism of the 20th century. It also is going to stay in repetoire for ever. There is no delay, which is perfect, with the tangible shapes that the solo violin digs into space. The violin achieves, before it leads into risky melodic flight, the point in which there is like a precarious orbit around the chamber orchestra in the spectacular first movement.Adès is able to reshape other composers' line of vision. That is why, the second movement and its beginning as a baroque channone is not a suprise. While the orchestra comes into view to move forward at a linear structure, the violin tears. Considering third movement, as for Adès, the orchestra follows stable cycles moving in harmony at different rates. Tension is also released softly in this part while the violin floats above the wide sky that is entirely free in the harmonic cycle of the orchestra.

\section{Movements}

\section{Rings}

Rings opens with energetically alternating D6 and G4s in the solo violin, supported thinly in the orchestra. The violin comes with a musical disorder of nearly constant semiquavers at the start of the 
piece. The instability can be heard as well in the very same part. This obviously has an influence on the orchestra. Unstable harmony in different orbist is one of the main features here. It is followed by impatient constant movement and restless arpeggios. Furthermore, harmonic sounds keep changing. Melody that begins to move from one magnetic-tonal attraction to another is both singable and recognisable. The violin is in dialogue with winds as the movement continues to twist. The soloist at this point flies above the orchestra with a tone which makes you feel like this is the moment of arrival. So, the musical planet exists and the orchestra can orbit. That is why, it is possible to say that Rings consists of instability and a system that is not that pure. It is like alive notes creating instability and making variations as dramatic falls bring a unique, colorful mode of harmonic progression.In other words, a particular pattern in which related movements and interactions are observable is displayed. There are, however, shifts that occur at a slow dimensions and Rings can be associated with the disorganized, curved paths of music (Fox). Nonetheless, it is certaint that it manages to include a process that is unified. Hence, combination of chromatic tones, unified process, and descents appear in the Rings.

\section{Paths}

A wider and a pleasant voyage can be found in the Paths section. It mostly depends on a warped chaconne and a repeating sequence of fourteen chords. The gravitational center is placed into the middle by Adès. The reason is that the slow movement is longer than the outer movements which create an effect in combination called as "triptych" by him. This movement is build on two large and very many small, independent cycles as it is slow. Those cycles, sometimes, overlap and are in a conflict while they rotate. The ritualistic power of the music is achieved with ceremonial-sounding cymbals (Conway). Interruptive punctuations, ferociously, increase the expressions of the soloists' so that it reaches a moving fluency in its successfull overlapping style. The violin descends by degrees to the bottom at the end of this movement while the music turns into a true resolution.

\section{Rounds}

The solo violin enters in Rounds and slow melody starts to fly like a satellite to the orchestra. In this part, full of energy, life, and enjoy can be visible. The wind of popular music can be slightly sensed in this section. The violin appeases with simple gestures of pureness. It is remained calm by cycles orbiting around. This stable cycle that is moving in harmony at different rates may be considered Rondo (May). In other words, opening theme, more or less, repeats itself with an alternative manner. The violin's semiquavers launch upwards which is a suprise hidden at the ending. Also a quite ending was composed by Adès as an distinct version.

\section{Irrational Bars - Additional Notation}

Although Adès uses a rhytmic process regarding his composing style, he also builds a tonaly complex music. A concerto refers to a dialogue between the soloist and the orchestra. Adès wants to establish a thin separation of the metric orbit of the soloist and the orchestra. Therefore, he uses highly cultivated rhytmic process. Here is an example of alternative notation made by him for the soloist.

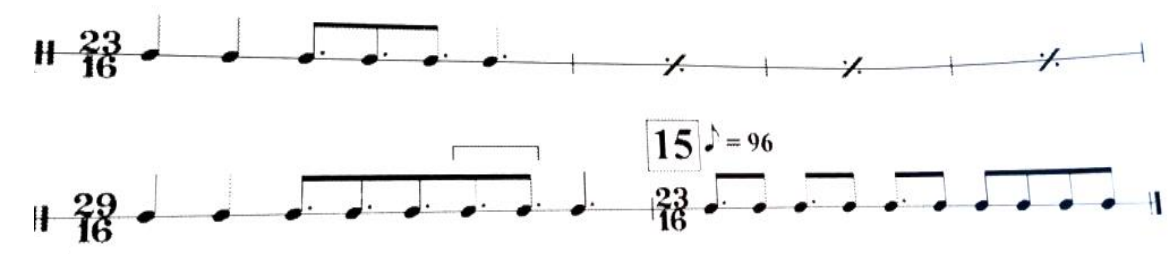

The special aspect of this notation is its original pride and inelegance. Notated part of triplets are fall under conventional bar. Formless meter that is recovered from the ensemble tracks the pattern. Here is an example for Adès' orchestral texture and the solo part. 

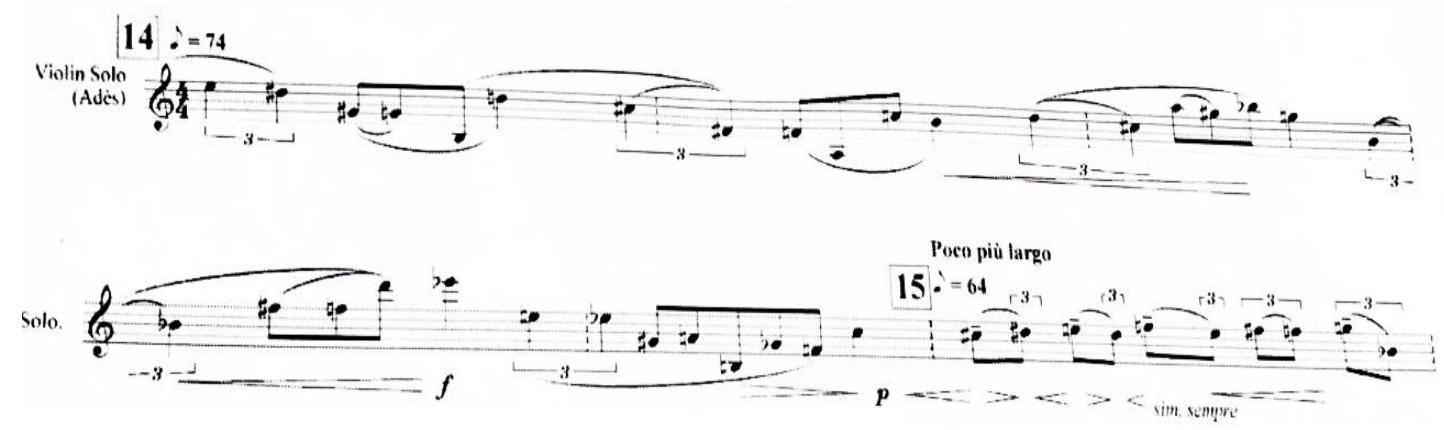

Repetitions and slow tempo in rhytm can be seen as a first sign. Pattern falls that note lenght is (a 1/6th crotchet) short of a full 4/4 bar, P9/42 (14). Gear alters among two meters and this shifts are caused by the alternative notation. Placing triplets can be a solution for this issue. This split notational method supports mentioned pride and in addition to that, no damage is done by alternatives gained from paradigm in Adès' own notational method.

\section{Similarities between Adès and Sibelius}

According to Augustin Hadelich, German violinist who born in Italy, Jean Sibelius's post-romantic violin concerto carries some aspects in common with Adès'. Adèscomposed his concerto almost a century later,2005, than Jean Sibelius.

Augustin Hadelich record an album with these two concertos. In album's booklet it is written that "The deep, rumbling timponi and low winds in the Sibelius bridge the gap to the Adès, a work which also explores the lowest depths of the sound spectrum, creating chasms over which the violinist performs a tightrope act"

The term centrifugal can be used for Adès Violin Concerto since everything placed is moving both around central point and out from central point.Moreover, as in the concerto of Sibelius, Adès' violion emerges at time to times like a lonely character on a space quest. Therefore, Hadelich claims that in this concerto he circles and swoops in the highest register of the violion.

\section{Conclusion}

The style, mode of this concerto might be considered neo-expressionist. Adès primary intension is not to abandon tonal base but instead to establish very infinite total universe. The Violion Concerto consists of three very special movements which are Rings, Paths, and Rounds. It is a triptych while the middle on is the largest. In the first movement Rings, Adès follows a pattern that always modulates. This section can be named as being overtune. The second movement, however, embody a linear and improvisational sort of structure. Beginning from the first chord of the violin, audience can clearly hear J.S. Bach's Chaccone. Adès provides an opportunity for the soloist in terms of display his/her abilities. The third and the last movement, the idea of a unified universe is more intensely heard motives in both violin and orchestra. The starting pattern of this section develops until the end of the concerto.Hence, Adès has managed to compose such a unique concerto. While it contains different orbits regarding both harmonical process and instruments, it is also unified.The notes, the features, the process itself, both descents and ascents, and much more are all important and they all function in this concerto. It is a kind of work that expresses it self in its own special way. 
International Journal of Scientific and Technological Research

ISSN 2422-8702 (Online), DOI: 10.7176/JSTR/7-03-03

Vol.7, No.3, 2021

\section{References}

Church, M. “Anthony Marwood: The Magic Violinist”. The Independent. (2006)

Conway, P. "Thomas Adés; Violin Concerto; Three Studies from Couperin; Overture, Waltz and Finale from Powder Her Face". Faber Music. (2010): 69-70.

Fox, D. "Multiple time-scales in Adès's rings [Elektronic version]". Perspectives of New Music.Vol. 52, No. 1. (2014): 28- 56.

Mangum, J. "Violin Concerto, Concentric Paths Op. 24". The Saint Paul Chamber Orchestra.Web. 12 Mar. 2016.

May, T. "Violin Concerto, Concentric Paths". About the Piece.Walt Disney Concert Hall.Web. 9 Mar. 2016.

Quinn, M. "Thomas Adés; Violin Concerto\&Tevot Review". BBC Review. (2010). Web. 9 Mar. 2016. 